fossa. The lesions involved the facial, vagus and hypoglossal nerves, on one side, together with some of the upper cervical motor nerves and some fibers of the opposite vagus. The spinal accessory nerve apparently escaped.

The provocative agent was a home-distilled "moonshine." Since the advent of prohibition and the consequent increase in the consumption and manufacture of improperly distilled liquors and synthetic concoctions, a new form of alcoholism has come to light, differing considerably from the classic type of days past. Its predilection for the ocular apparatus and its tendency toward strange psychoses are among the more recently observed manifestations. Our case demonstrated the extremely toxic effects of these beverages on nerve cells, as manifested by the multiple neuritis as well as the mental derangement.

History.-C. W., man, aged 43, a woodworker, was brought in suffering with difficult respiration of about eight hours' duration. He stated that the attack began the night before, with a choking sensation, and that he had never suffered an attack of this kind before; nor could he state what brought in on. The dyspnea was chiefly inspiratory.

Examination.-The preliminary examination revealed an emphysema and piping râles throughout the chest. A provisional diagnosis of asthma was made. A little later a laryngeal examination was made. It was found that the vocal cords were in complete adduction, and, except for a slight wavering of the right cord, failed to abduct on inspiration. There was a slight amount of edema of the arytenoids and the aryepiglottic folds.

The cyanosis and stridor, though marked, were not sufficient to necessitate immediate tracheotomy. The following day, the dyspnea was still pronounced, and the patient complained of headache, cough, and nausea, which were later followed by vomiting. These symptoms persisted, and, in addition, the patient became irrational and boisterous, and tried to get through a locked door leading to the fire escape. Restraints were applied. He continued to be very restless and noisy, talking loudly and irrationally. The pulse was weak and thready, ranging from 130 to 156 , and the respirations were correspondingly rapid (48 to 54 ). His expression was anxious, and he perspired freely. Cyanosis, though present, was not threatening. In addition, the examination disclosed: (1) paresis of the right side of the face; (2) paresis of the right half of the tongue; (3) inability to elevate the chest (paresis of the accessory muscles of respiration; the pectoralis minor, serrati and scaleni); (4) increased knee jerks; (5) doubtful Babinski on the left side; (6) normal pupils and fundi.

The multiplicity of symptoms suggested cerebrospinal syphilis, which diagnosis, however, was later ruled out through the subsequent course of the case and the negative Wassermann reactions on both blood and spinal fluid.

The leukocytes numbered 9,800 ; the urine was negative for albumin, sugar and casts. The following day the patient was improved. Breathing was easier; he was more rational, quieter and the pulse was slower (pulse, 118; respiration, 24). Examination of the larynx revealed the left cord immobile in adduction; the right cord abducting slightly on inspiration; the epiglottis and arytenoids somewhat edematons.

At this time, by close questioning, we obtained the information that the patient had for some time past been imbibing freely of a home-made alcoholic distillate which was being manufactured in the neighborhood, and that just previous to the onset of the attack he had obtained several bottles that had recently been made. Nothing definite could be ascertained as to the exact nature of the concoction, except that it was almost white and was "very strong."

A roentgenogram of the chest at this time proved negative. Course.-The paresis of the face, tongue and chest muscles disappeared within forty-eight hours, and the laryngeal condition showed progressive improvement. Examination on the nineteenth day, under direct laryngoscopy (local anes- thesia), revealed the right cord moving freely, both in abduction and adduction; the left cord was still in adduction and moved but slightly on deep inspiration. There were slight pachydermic masses on both cords and the interarytenoid space, but no edema, tumor or alcer.

The patient returned to the hospital for observation at weekly intervals for two months and showed a general improvement, gaining 20 pounds $(9 \mathrm{~kg}$.) in weight.

Examination, three months after the attack, demonstrated the right cord completely recovered and the left one almost in the cadaver position, although showing some slight motion on phonation. It appears that the paralysis of the recurrent nerve was almost complete on this side, having gone through the cycle in accordance with Semon's law, beginning with the abductors and ending with the adductors.

The voice is, nevertheless, very good, as the right vocal cord during phonation overrides the median line and opposes the left cord in the oblique position.

\section{RELATIVE VALUE OF SACHS-GEORGI AND WASSERMANN REACTIONS IN DIAGNOSIS OF SYPHILIS}

\author{
AN ANALYSIS OF ONE THOUSAND SEVEN \\ HUNDRED AND FORTY-EIGHT \\ PARALLEL TESTS * \\ CHARLES F. CRAIG, M.D. \\ Lieutenant Colonel, Medical Corps, U. S. Army \\ AND \\ WILLIAM C. WILLIAMS \\ Captain, Medical Administrative Corps, U. S. Army \\ WASHINGTON, D. C.
}

An enormous literature has accumulated regarding the relative merits of the Sachs-Georgi and the Wassermann tests in the diagnosis of syphilis, a literature that is so filled with contradictions that an unprejudiced reader is utterly at a loss in evaluating the relative accuracy of the two tests. Some observers claim for the Sachs-Georgi reaction a greater specific value in the diagnosis of the disease than is possessed by the Wassermann reaction, while others conclude that the Sachs-Georgi test is less accurate or absolutely unreliable.

In view of the discordant results recorded in the literature with the Sachs-Georgi test, we believed that it would be of some value to compare the results obtained with the two tests on routine specimens of blood sent to the laboratories of the Army Medical School for the Wassermann test. This work has covered a period of nearly one year and has been very carefully conducted, the Wassermann tests being made and read by one of us (W. C. W.), while the SachsGeorgi tests were made and read by the other (C. F. C.). In this way all leaning toward forcing an agreement between the results of the tests, which might unconsciously be brought about when a single person makes and reads both tests, was avoided and an absolutely unprejudiced reading of both was possible, as neither of us knew at the time of reading his tests what result had been obtained with the comparative test.

From our experience we are positive that a greater agreement between the two tests would have been obtained had one observer made all of the readings for both, but we are also positive that the data so obtained would have been very unreliable, as many of the weak Sachs-Georgi reactions would have been read

\footnotetext{
* From the Division of Laboratories, Army Medical School.
} 
as positive in order that the result might agree with the result of the Wassermann test, despite the fact that just as strong Sachs-Georgi reactions were frequently noted in nonsyphilitic cases. The very high percentage of agreements between the results of the Sachs-Georgi and the Wassermann tests recorded in the literature by some observers were perhaps due to the fact that readings of the two tests were made by a single observer who had a knowledge of the results of both and who, in comparing the results, unconsciously strove for an agreement in the doubtful cases. At any rate, we feel sure that in comparative research of this character it is most important that the results of the tests under comparison be read by independent observers, as otherwise it is practically impossible to avoid errors due to a desire to force agreements where none really exist. For this reason we believe that our results are more truly representative of the real relative value of the two tests than many that have been published in the literature.

\section{TECH NIC}

The technic employed in making the Sachs-Georgi tests was that recommended by Sachs and Georgi, ${ }^{1}$ with slight variations in the mixing of the antigen and salt solution, which will be referred to later. The antigen employed consisted of a 20 per cent. alcoholic extract of beef heart muscle fortified by the addition of cholesterin. The amount of cholesterin to be added in order to secure the best results was determined by carefully weighing out different amounts and adding them to 10 c.c. of the extract in such a manner as to make the different portions of the antigenic extract contain $0.2,0.3,0.4,0.5$ and 0.6 per cent. of cholesterin. These portions of cholesterinized extract were then titrated with known positive and negative serums and the portion of extract giving the best results was selected for use in the test. The percentage of cholesterin to be added to the alcoholic extract of beef heart was found to vary slightly with different extracts, but was generally either 0.4 or 0.5 per cent.

The cholesterinized extract before use in the test was diluted with 5 parts of physiologic sodium chlorid solution. The method by which this dilution is made is of immense importance as regards the results of the test, as will be shown later.

The serums to be tested were inactivated at $55 \mathrm{C}$. for one-half hour in the water bath and diluted with 9 parts of physiologic sodium chlorid solution, after which 5 parts of the diluted antigen were added and the whole mixture incubated at $37 \mathrm{C}$. for from eighteen to twenty-four hours. In our tests each test tube contained 0.9 c.c. of physiologic sodium chlorid solution, 0.1 c.c. of the serum to be tested and 0.5 c.c. of the antigenic suspension.

The technic used in the Wassermann tests was the modification devised by one of us (C. F. C.), which is used in the routine work of this character at the Army Medical School. In this modification a human hemolytic system and a cholesterinized antigen are employed. The exact technic has been described in detail elsewhere. ${ }^{2}$

Terms Employed in Reading the Tests.-In reading and recording the results of both the Sachs-Georgi and the Wassermann tests the following terms were employed: Double plus $(+t)$, plus $(t)$, plus-

1. Sachs, H., and Georgi, W.: Med. Klin. 14: 805, 1918.

2. Craig, C. F.: The Wassermann Test, Ed. 2, St. Louis, 1921, pp. $99-116$. minus $(+-)$, and negative $(-)$. The term double plus indicates complete flocculation with the SachsGeorgi test and complete inhibition of hemolysis with the Wassermann test. The term plus indicates marked flocculation with the Sachs-Georgi test and anything between complete inhibition of hemolysis and 50 per cent. of hemolysis with the Wassermann test. The term plus-minus indicates very slight flocculation with the Sachs-Georgi test and anything between 50 per cent. of hemolysis and complete hemolysis; while the term negative indicates no flocculation with the Sachs-Georgi test and complete hemolysis with the Wassermann test.

The Sachs-Georgi test owes its origin to the observation that when a stitable amount of a cholesterinized alcoholic extract of a human or beef heart is added to the diluted blood serum of a patient suffering with syphilis, a characteristic flocculation occurs in the mixture, either immediately or after incubation, and the value of the test depends entirely on the degree of flocculation noted in the serum tested. In our work the utmost care was exercised to determine the presence or absence of this phenomenon in the serums tested and it early became evident that if we depended on reading the tests with the naked eye we would miss many positive reactions. For this reason we have read the end reactions of the Sachs-Georgi test with a low power hand lens against a dark background. We found that considerable practice was necessary before some of the positive reactions with this test could be differentiated from the appearance caused in some serums by the formation of certain precipitates and that even with the greatest care it was sometimes impossible to be sure of the presence or absence of flocculation. From our experience in this respect, we believe that the use of this test should be restricted to those: well trained in its technic and interpretation, for otherwise serious errors in its diagnostic use will certainly occur very frequently.

The difficulty of reading the partial reactions and of interpreting their meaning is great with the SachsGeorgi test, and we soon found that any reaction with this test below a plus reaction was absolutely valueless in diagnosis. Thus all plus-minus reactions were worthless, as a slight amount of flocculation occurred just as frequently in nonsyphilitic serums as in those known to have been taken from syphilitic patients. In this report, it should be understood that when the Sachs-Georgi reaction is called positive, it means that at least a plus reaction was obtained in the serum examined. All plus and double plus reactions with the Sachs-Georgi test were considered as positive reactions in our work, and all plus-minus and negative reactions were considered negative. With the Wassermann test, only double plus reactions were considered as positive reactions, but the plus reactions with this test were included with the positive reactions in estimating the total agreements with the two tests.

In all, 1,748 serums were tested with both tests, and at the same time. The tests may be divided into two groups, as regards the results obtained: Group 1, in which the antigenic suspension was used within from ten to fifteen minutes after adding the saline solution to the antigenic extract, and Group 2, in which the salt solution was added to the antigenic extract and the mixture allowed to stand for two hours before use. As will be noted from a study of the results obtained, very much better results were secured when the anti- 
gen was diluted and allowed to stand for two hours before use. In Group 1, 748 serums were tested with both tests, while in Group 2, 1,000 serums were tested.

The fact that allowing the antigenic mixture to stand for two hours after adding the salt solution greatly increases the diagnostic value of the Sachs-Georgi test is well shown in our results and illustrates only one of the many factors that either increase or decrease its delicacy and thus render this test one that is unstable and liable to great variations in results in different laboratories. Thus the mere method of adding the salt solution to the antigenic extract is most important, for, if it is added rapidly, less accurate results and a smaller number of positive reactions are obtained than when it is added slowly, even drop by drop. The exact mechanism of this reaction is yet unknown, but it undoubtedly depends in large measure on the surface tension of the mixtures and this is markedly influenced by causes that are often beyond the control of the investigator.

We believe that the results that we obtained in the serums included in Group 2, taking into consideration the fact that the readings were absolutely uninfluenced by a foreknowledge of the results obtained with the comparative test, represent what may be expected of the Sachs-Georgi test when it is carefully made under the best possible conditions, and that the results in Group 1 represent what may be expected of it when some little understood factor, as the time that the antigenic extract and the salt solution should remain in contact, is active. In this way, the greatly divergent results of this test as reported in the literature may be partially explained.

RESULTS OF PARALLEL TESTS IN GROUP 1

In Group 1 are included the serums tested with the Sachs-Georgi test in which the antigen was diluted with 5 parts of physiologic sodium chlorid solution $(0.85$ per cent.) and the mixture used within fifteen minutes after the salt solution had been added. In this group, 748 serums were tested with both the Sachs-Georgi and the Wassermann tests with the following results: total number of parallel tests made, 748 ; total number of positive $(++)$ Wassermann reactions, eightyeight, or 11.7 per cent. ; and total number of positive Sachs-Georgi reactions, fifty-three, or 7.0 per cent.

In addition to the positive or double-plus Wassermann reactions mentioned, there were sixty-two plus Wassermann reactions, or 8.2 per cent. of the total number of serums tested, which should, without doubt, be included in the positive Wassermann tests, as they were obtained in very early or treated syphilitic infections. If these are added to the double-plus Wassermann reactions, we have a total of 150 positive Wassermann tests, or nearly 20 per cent. of the total number of serums tested, as compared with only 52 positive Sachs-Georgi reactions, or 7 per cent. of the total number of serums tested.

However, the chief interest in these parallel tests with the two reactions is concerned with the number of agreements in results. Taking into consideration only the double plus Wassermann reactions and the double plus and plus Sachs-Georgi reactions, there was an agreement in results in 596 serums, or 79.6 per cent. of the total serums tested. If to these figures are added those serums in which a single plus Wassermann reaction was obtained and a positive Sachs-Georgi test, the number of agreements are increased to 602 , or 80.4 per cent of the total serums tested.
This is a fair average percentage of total agreements between the two tests as shown in the literature; but, when one examines the total number of agreements in the positive and negative reactions, our results are very disappointing as regards the value of the Sachs-Georgi test in the diagnosis of syphilis. Thus, in only twentyfive of the eighty-eight double plus Wassermann serums did the Sachs-Georgi test give a positive reaction or a total agreement of only 28.4 per cent. In other words, sixty-three serums, or 71.5 per cent. of the double plus Wassermann cases, were absolutely missed by the Sachs-Georgi test.

On the other hand, there were twenty-two serums in which the Sachs-Georgi test gave a positive result and in which the Wassermann test was doubtful or negative. These serums constituted 41.4 per cent. of the total positive Sachs-Georgi reactions so that it may be stated that 41.4 per cent. of the total positive SachsGeorgi reactions were not confirmed by the Wassermann test. If this fact indicated that this number of syphilitic cases were missed by the Wassermann test, it would speak very favorably for the superior delicacy of the Sachs-Georgi reaction; but, as will be later observed, such an interpretation cannot justly be placed on these disagreements in results.

As regards the negative reactions obtained with both tests, there was a very close agreement, no less than 571 of the serums giving a negative reaction with both tests. Thus, of the 593 serums that gave a negative reaction with the Wassermann test no less than 571 also gave a negative reaction with the Sachs-Georgi test or an agreement percentage of 96.3 .

From the results given it is evident that in this groum of parallel tests, the Sachs-Georgi reaction proved to be of very much less value in the diagnosis of syphilis than the Wassermann reaction and that it was untrustworthy in that no less than 41.4 per cent. of the comparatively few positive reactions obtained with it were not confirmed by the Wassermann reaction, or, as will be shown later, by the clinical symptoms or history of the patients from whom the blood serum was obtained.

RESUlTS OF THE PARALLEL TESTS IN GROUP 2

While the work on the tests in Group 1 was being conducted, it was noted that an antigenic extract to which the physiologic sodium chlorid solution had been added two hours before use gave a much higher percentage of positive results with the Sachs-Georgi test than the same antigenic extract to which the saline solution had been added from ten to fifteen minutes before use. This phenomenon was investigated by adding the saline solution to the antigenic extract and waiting for varying periods of time before using the mixture in the test, and it was found that the maximum of positive results was obtained when an interval of two hours was allowed to elapse after the addition of the salt solution before using the mixture as an antigen.

All of the serums included in Group 2 were tested simultaneously by both the Sachs-Georgi and the Wassermann tests. The antigen in the Sachs-Georgi test was mixed in the manner above described, and, if the results obtained with this test are compared with those obtained in Group 1, it is at once obvious that they are better than those obtained with the freshly mixed antigen.

In Group 2, 1,000 serums were tested, with the following results: total number of parallel tests made 1,000 ; total number of positive $(++)$ Wassermann 
reactions, 156 , or 15.6 per cent.; and total number of positive Sachs-Georgi reactions, 181, or 18.1 per cent.

In addition to the positive, or double plus, Wassermann reactions, there were fifty-three plus Wassermann reactions, or 5.3 per cent. of the total number of serums tested which should be included in the positive Wassermann reactions, as most of them were in known treated syphilitic infections. If these are added to the double plus Wassermann reactions, the total positive Wassermann reactions equal 209, or 20.9 per cent., as compared with 181 positive Sachs-Georgi reactions, or 18.1 per cent. of the total serums tested.

As regards the number of agreements between the tests in this group of cases, taking into consideration only the double plus Wassermann reactions and the plus and double plus Sachs-Georgi reactions, there was an agreement in results in 832 cases, or 83.2 per cent. of the total serums tested, while if to these figures are added the plus Wassermann reactions in which the

TABLE 1.-COMPARATIVE RESULTS OF THE SACHS-GEORGI AND THE WASSERMANN TESTS IN 1,748 CASES

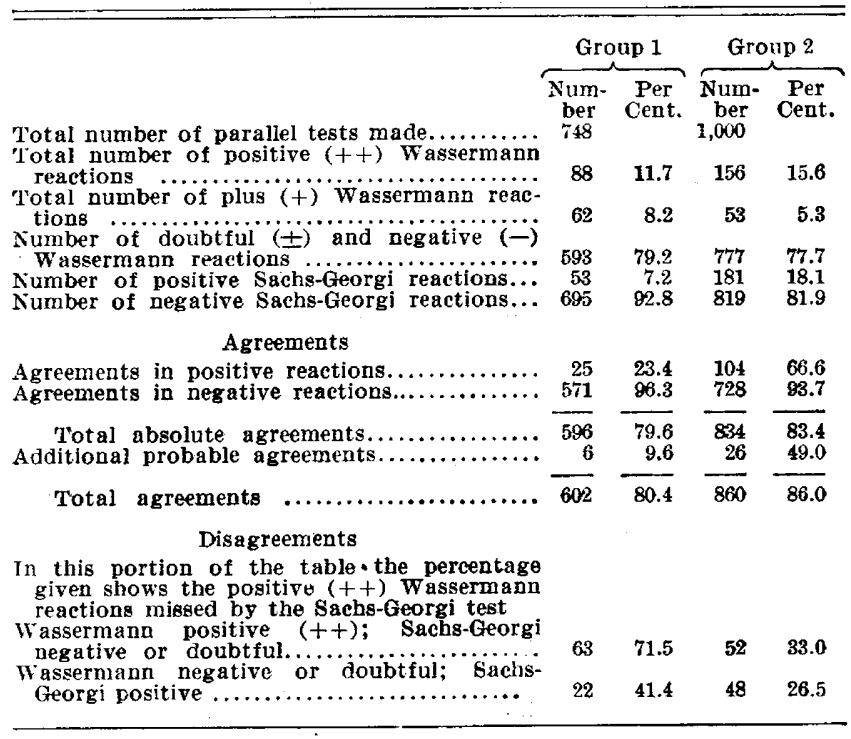

Sachs-Georgi test was positive, twenty-six in number, the two tests agreed in their results in 858 cases, or 85.8 per cent.

This percentage of total agreements between the two tests is about that reported by most observers; but here, as in Group 1, a careful examination of the number of agreements between the positive and negative reactions with the two tests proves very disappointing so far as the practical value of the Sachs-Georgi test in the diagnosis of syphilis is concerned. Thus, in only 104 of the double plus Wassermann reactions, 156 in number, did the Sachs-Georgi test give a positive reaction, more than 33 per cent. of the cases giving a positive Wassermann reaction being missed by the Sachs-Georgi test. There were forty-eight serums in which the Wassermann test was negative or doubtful and which gave a positive reaction with the Sachs-Georgi test. These serums comprised 26.5 per cent. of the total number of serums giving a positive Sachs-Georgi reaction, so that it may be stated that 26.5 per cent. of the positive Sachs-Georgi tests could not be confirmed by the Wassermann test, and an inquiry into the history of the patients from whom these serums were obtained demonstrated that neither the history nor the symptoms present at the time of the blood examination indicated syphilitic infection.

In this group of serums, the number of agreements between the negative tests was high, no less than 728 of the total 777 serums giving a negative result with the Wassermann test, or a total negative agreement of 93.7 per cent.

The accompanying table gives the results that we obtained with the Wassermann and Sachs-Georgi tests in both Group 1 and Group 2 and well illustrates the marked differences in the results with the Sachs-Georgi test, produced by the delay of two hours in using the antigenic mixture after the salt solution was added.

\section{ANALYSIS OF DISAGREEMENTS BETWEEN THE TWO TESTS}

In view of the importance of ascertaining the real, practical relative value of the Sachs-Georgi and the Wassermann tests in the diagnosis of syphilis, a careful study was made of the cases in which the two tests gave divergent results. These cases may be divided into those giving a double plus or positive Wassermann reaction and a negative Sachs-Georgi reaction and those giving a positive Sachs-Georgi reaction and a negative Wassermann reaction. Only the disagreements observed in Group 2 were considered, as it was in this group that the best results were obtained with the Sachs-Georgi test and it was our desire to give the latter test the fairest trial possible before reaching a conclusion as to its value in diagnosis, as compared with the Wassermann test.

Cases with a Double Plus Wassermann Reaction and a Negative Sachs-Georgi Reaction.-There were fiftytwo specimens of blood serum from as many patients that gave a positive Wassermann reaction and a negative Sachs-Georgi reaction. A very careful inquiry was made into the antecedents, history and symptoms of these patients, with the following results:

Of the fifty-two cases, forty-five, or 86.9 per cent., were either known or probable cases of syphilis presenting suspicious histories or symptoms of the disease. In four cases, or 7.6 per cent., no history of syphilis could be obtained, and, save for an optic atrophy in one case, "these patients presented no evidences of syphilis. However, in three of these cases, the positive Wassermann reaction was again obtained at a later date, and it is probable that these patients were really suffering from a latent syphilitic infection. These cases are not included in the forty-five known infections just mentioned. In three cases, no information could be obtained.

The investigation of the cases in which the Wassermann test was positive and the Sachs-Georgi negative demonstrated that forty-five of a total of 156 patients with a positive Wassermann reaction and known to be syphilitic gave a negative reaction with the SachsGeorgi test. In other words, 28.8 per cent. of the known syphilitic patients would have remained undiagnosed, so far as a test of their blood serum is concerned, had we depended on the Sachs-Georgi reaction in diagnosis.

Cases with a Negative Wassermann Reaction and a Positive Sachs-Georgi Reaction.-There were fortyeight specimens of blood serum from as many individuals that gave a negative or doubtful Wassermann reaction and a positive Sachs-Georgi reaction. These specimens constituted 26.5 per cent. of the total number of serums giving a positive Sachs-Creorgi reaction. 
The great importance of ascertaining whether the individuals from whom the blood serum was obtained were syphilitic is obvious, and a very careful and searching inquiry was made into the history and symptoms of each case, with the following results:

Of the forty-eight individuals giving a positive Sachs-Georgi and a negative Wassermann reaction, no less than forty, or 83.3 per cent., gave no history of syphilitic infection and presented no signs or symptoms of the disease. Five of the individuals were known syphilitic patients and in three of the cases no information could be obtained.

Our investigation, therefore, demonstrated that forty of a total of 181 positive Sachs-Georgi reactions, or 22.1 per cent., were undoubtedly false reactions and were not confirmed by the Wassermann test or the history or symptoms of the patients from whom the blood was obtained, and that five of the total 181 positive Sachs-Georgi reactions, or 2.7 per cent., were in known cases of syphilis in which the Wassermann test was doubtful or negative. In three of these cases, the Wassermann test gave a plus-minus reaction and their history showed them to be old latent infections.

\section{VARIATIONS IN THE RESULTS OF THE SACHS- GEORGI TEST}

In our experience, a very noticeable characteristic of the Sachs-Georgi reaction was its variation in results on specimens of blood serum obtained from the same patient but examined at different times. A few examples of this variability are given in the subjoined case reports.

\section{REPORT OF CASES}

CASE 1.-P. S., a man, was in the hospital for a mild respiratory infection. His blood serum gave a negative Wassermann reaction and a very strongly positive Sachs-Georgi reaction when first examined. There was a negative history as to syphilis, and no clinical symptoms. Three weeks later, after discharge from hospital, his blood serum still gave a negative Wassermann reaction, but this time a negative Sachs-Georgi reaction was obtained.

CASE 2.-Mrs. W. had no history or evidence of syphilis. The blood serum gave a double plus Wassermann reaction and a negative Sachs-Georgi reaction. The second Wassermann test, ten days later, gave a double plus Wassermann reaction and a negative Sachs-Georgi reaction. The third Wassermann test, three weeks later, gave a strong plus Wassermann reaction and a strongly positive Sachs-Georgi reaction.

CASE 3.-R. E. L. had a known case of syphilitic infection. The blood serum of this patient gave, at different times, two double plus Wassermann reactions and two negative SachsGeorgi reactions. A third test resulted in a very strong plus Wassermann reaction and a strongly positive Sachs-Georgi reaction.

CASE 4.-The first test on the blood serum in this patient gave a double plus Wassermann reaction and a plus SachsGeorgi reaction. There was a history of an abortion and a stillbirth, probably syphilitic in origin. A second test made, twelve days later, resulted in a double plus Wassermann reaction and a clean cut negative Sachs-Georgi reaction.

The cases just cited are only a few examples of the many variations that were obtained with the SachsGeorgi reaction when several tests were made on the blood serum of the same individual at different times, variations so marked that they could not be explained by any normal variation in the reacting substances in the serum examined. Indeed, if repeated tests had been made on the entire number of individuals examined, it is quite possible that the percentage of variations in the results obtained with this test would have been so pro- nounced and so confusing as to cause very grave doubt as to the real diagnostic value of the test in any one case. This is a phase of the subject that should receive further investigation.

\section{SUMMARY AND CONCLTSIONS}

1. The results of parallel Sachs-Georgi and Wassermann reactions on 1,748 specimens of blood serum from as many individuals gave a total agreement in results of 85.5 per cent.

2. The disagreements, however, were most serious, no less than 33.3 per cent. of the positive Wassermann reactions being missed by the Sachs-Georgi test in known infections with syphilis, while 26.5 per cent. of the positive Sachs-Georgi reactions were not confirmed by the Wassermann test nor by clinical findings.

3. These disagreements in tests performed under ideal conditions for comparison, by two separate investigators, were so radical in character and so important from a diagnostic standpoint, that we do not believe that the Sachs-Georgi reaction alone should be relied on as a diagnostic method for syphilis under any conditions.

4. The difficulty of reading slight Sachs-Georgi reactions and differentiating them from precipitation due to other causes in the serum tested is so great, in many instances, that no reliable conclusion can be reached, thus leaving the test open to so much individual interpretation that it destroys the scientific value of the results. The widely divergent results with the test reported in the literature are conclusive proof of this statement.

5. The Sachs-Georgi test, using the technic recommended by the originators, or slight modifications of this technic, is an interesting reaction from a theoretical and speculative standpoint; but it is greatly inferior, in its practical application to the diagnosis of syphilis, to the Wassermann test or any of its accepted modifications.

6. The Sachs-Georgi test should never be used to the exclusion of the Wassermann test in the diagnosis of syphilis, and a positive result with the Sachs-Georgi test should always be checked up by a Wassermann test.

Effect of Altitude on Human Life.-The eight investigators, five of them Harvard Medical School graduates and the other three British, who sailed for Peru last November to make their headquarters at Cerro de Pasco (altitude 14,200 feet, in the Andes) for the purpose of studying the changes in the heart, circulation, respiration, and chemical composition of the blood which enable the residents to live in comfort at an altitude at which most people could hardly exist on account of rarity of the air, have made a report. An improvised laboratory was taken in the baggage car, and blood tests of the inhabitants were taken, by which it was shown that these people live under conditions in which blood does not contain the usual quantity of oxygen. They are able to carry heavy burdens up sharp inclines at this altitude without exhaustion. The blood tests taken gave some insight into the chemical changes which take place in the blood to make this possible. The members of the expedition were: Dr. Alfred C. Redfield, Arlie B. Bock, Henry S. Forbes, Boston; Carl A. L. Binger, George Harrop, New York; Prof. Joseph Barcroft, Cambridge University, England, who organized the expedition; Prof. J. G. Meakins, Edinburgh University, Scotland, and Dr. Doggart of Kings College, Cambridge. The expedition was financed by Kings College, Cambridge, Royal Society of London, Carnegie Foundation, Rockefeller Institute, the Presbyterian Hospital, New York, University of Toronto, Harvard Medical School, and certain private individuals. 